\title{
ESTADO ACTUAL DE LA REACCION DE GALLI-MAININI
}

\author{
Doctor Gilberto Angel M.*
}

Desde el año de 1948 en que Carlos Galli Mainini hizo su primera publicación (1) relacionada con su reacción, centenares de artículos han sido publicados en todo el mundo; unos encontrando en élla el mejor test como diagnóstico precoz del embarazo, otros manifestando su escepticismo por haber obtenido resultados discordantes y algunos no muy partidarios con el factor sapo, inestable en las estaciones, con variedades regiona!es de cada país y de diferente susceptibilidad a la liberación espermática. Por haber tenido una estrecha amistad con el autor de la reacción hasta su temprano fallecimiento, la curiosidad de revisar periódicamente las publicaciones en Excerpta Médica de Obstetricia y Ginecología (Años 1949-1965) donde hay numerosas publicaciones de trabajos realizados en la India, Japón, Rusia, China, Australia, Italia, etc., etc., sobre el tema que nos ocupa y una experiencia personal con un número de reacciones verificadas superior a 12.000 , creo de alguna utilidad sintetizar lo que de élla podemos esperar y sus diferentes interpretaciones, hoy en día verdaderamen-

* Departamento de Morfología. Universidad del Valle, Cali. te sedimentadas por los años transcurridos desde su descubrimiento.

\section{Precocidad de la reacción}

En la especie humana el óvulo fecundado en el tercio externo de la trompa, llega a la cavidad uterina y alcanza su madurez de implantación alrededor de 6 días después de la fecundación (Hertig y Rock) (2). Las vellosidades coriales por intermedio de su capa trofoblástica inician la producción de gonadotrofinas entre el 13 y 19 días después de la implantación (Smith y Randall) que llevadas por la sangre, pasan al suero sanguíneo, líquido cefalorraquídeo, saliva, calostro etc., para ser finalmente eliminadas por la orina. Si consideramos el tiempo de implantación y el que transcurre para iniciar la producción de gonadotrofinas tenemos 25 días, tiempo en el cual la reacción es correctamente positiva en el 99,9\% de los casos. Si la fecundación se inició en una ovulación precoz ( 5 a 7 días) podemos obtener resultados correctamente positivos a los 2 o 4 días de amenorrea; si lo fue en la mitad del ciclo, a los 15 días de amenorrea, pero si fué en ovulación tardía sólo se va a positivizar entre los 25 y 30 días de 
amenorrea cuando la reacción siempre tiene todo su valor.

\section{Factor sapo en la reacción}

En nuestro Departamento del Valle que podemos considerar como la fuente de producción de los Batracios, por ser el sitio de abastecimiento principal para el resto del país, encontramos las variedades Arenarum Hansel y Marinus, igualmente receptivos y con las mismas características de los que se encuentran en Argentina y Brasil. En Estados Unidos predomina el tipo Americanus (3), susceptible de dar falsas reacciones positivas; en Alemania, el tipo Viridis (4) nunca ha dado falsos positivos pero sí da falsos negativos sobre todo en invierno; en Londres, el tipo British (5) de una susceptibilidad extraordinaria da reacciones en el $60 \%$ de los casos en una hora, sin falsos positivos; en el Japón, el tipo Ishida con una positividad verdadera del $99,4 \%$, pero siempre que sea con orina concentrada (6). El factor sapo es el causante de conceptos tan diversos sobre la reacción, pues mientras nosotros encontramos todas sus ventajas, con la imposibilidad, en nuestro medio, de las falsas positivas, en otros países la variedad del animal no les permite verificar la exactitud descrita en algunas publicaciones. En Rusia (7), experimentalmente, han logrado producir espermaturia en los batracios seccionándoles los lóbulos ópticos anteriores, con la debida relajación de los centros medulares e inhibición de los altos centros nerviosos, explicando así la espermaturia en los sapos flacos y caquéc- ticos con más de tres meses de cautiverio.

La variedad nuestra no es susceptib'e de dar falsos reacciones positivas, pero sí negativas falsas en los siguientes casos: Cuando no han alcanzado su completa madurez sexual que es cuando pesa entre 50 y 70 grms. En los casos de alto parasitismo intestinal, predominando en ellos las larvas de Strongiloides y el Balantidium, y, cuando han tenido un cautiverio prolongado por la nula alimentación que aceptan. No son raros los casos de obtener en una reacción, con unos animales, resultados positivos y con otros, negativos, encontrándose, en los últimos, granulomas gigantes con diversos tipos de micosis, implantados en forma peri-renal. Por eso toda reacción que trate de dar el máximo de seguridad debe contar con animales de corto cautiverio y en un número no inferior a 4 animales por reacción.

\section{Factor orina y suero en la reacción}

Fisiológicamente en la mujer embarazada la gonadotrofina coriónica circula en el torrente sanguíneo y es eliminada por la orina. No existe ningún procedimiento que permita afirmar si en el momento de la toma sanguínea la concentración hormonal sea suficiente para hacer reaccionar el animal, si dicho nivel es bajo momentáneamente, obtendremos falsas reacciones negativas. En cambio la orina correspondiente a la primera micción matinal, teóricamente nos asegura una buena cantidad de gonadotrofina en dosis muy superior a la que necesita el sapo para reaccionar. Si la orina tiene una baja densi- 
dad, por dilución provocada al ingerir bastantes líquidos, también produce falsas reacciones negativas. $\mathrm{Si}$ bien es cierto que obtenemos reacciones positivas con muestras de orina tomadas en cualquier tiempo, la máxima seguridad la tenemos con la primera orina matinal, muestra ideal por su concentración, facilidad de obtenerla y brindarnos un tiempo prolongado durante el día para observar los resultados. No tiene ninguna aplicación práctica la determinación simultánea en orina y suero, dependiendo los resultados del momento de la toma y técnica empleada, fisiológicamente no existe ninguna razón para que sea positiva en una muestra y negativa en la otra, si se verifica con los cuidados y precauciones respectivas.

\section{Casos en que es preferible el suero}

Si la paciente está sangrando y no se toma la orina por cateterismo, es frecuente la muerte de los animales, por choque anafiláctico provocado por la substancia que se inyecta. En estos casos es preferible inyectar suero, con la desventaja de inyectar menor número de animales pues $20 \mathrm{cc}$ de sangre difícilmente nos proporciona una cantidad suficiente para los 4 animales. Con frecuencia relativamente baja, hay pacientes, que sin causa conocida, matan los animales con orina que no presenta ninguna alteración especial, pero en la gran mayoría de los casos, la muerte provocada obedece a contaminación del recipiente donde se ha recolectado, por substancias letales para el animal. Recibiendo la orina en frascos estériles, son excepcionales estos casos.

\section{La reacción en el feto muerto}

Una de las características de la reacción de Galli, es su precocidad en poner de manifiesto la gonadotrofina coriónica, pero normalmente en el último trimestre del embarazo se hace negativa en un $15 \%$ de las pacientes. Al morir el feto, se inicia la involución hormonal de la placenta y su nivel gonadotrófico decrece paulatinamente, pero el factor inicialmente considerado y el hecho de que no siempre existe una estrecha relación entre la muerte del feto y el cese activo placentario, el dato de Positiva o Negativa en sospechas de feto muerto, debe mirarse con reservas. También debe considerarse el hecho de que en la mola y coriocarcinoma donde no existe feto, la reacción es francamente positiva por el nivel gonadotrófico existente. En consideración a lo anterior, su valor en estos casos es muy relativo, y de poca ayuda a la clínica, en el último trimestre del embarazo, pero con un poco de utilidad en el comienzo del mismo, si antes existía una reacción positiva.

\section{En los tumores testiculares}

En las reacciones de Aschheim-Zondek y Friedman (8) la eliminación de gonadotrofinas relacionada con la destrucción de las células intersticiales, dan títulos cuando todavía no se han iniciado las metástasis, capaces de hacer reaccionar a las ratas y conejas. En el teratoma tipo adulto se eliminan cerca de 500 unidades rata, en el seminoma entre 2.000 y 10.000 U. R. y en el coriocarcinoma niveles superiores a las 10.000 U. R. Estas dosis son insufi- 
cientes para hacer reaccionar al sapo $\mathrm{y}$ por lo tanto no tiene ninguna utilidad en estas lesiones.

\section{Reacción de Galli y tranquilizantes}

En 1959 Hodgson (9) y Brillhart (10) publicaron sus experiencias de falsas reacciones positivas en hombres y mujeres que habían tomado promazina, prochlorperazine, perphenazine (grupo de las phenothiazine y derivados). En nuestro medio el doctor Miguel Bueno (11) realizó en el Hospital Psiquiátrico de San Isidro un trabajo con pacientes de ambos sexos que habían recibido diariamente dosis de phenothiazine y derivados entre $150 \mathrm{y}$ 800 mgrs, usando los sapos de la región, Bufo arenarum, en 103 pacientes (54 mujeres y 49 hombres) y 81 tests en 59 pacientes ( 34 mujeres y 25 hombres) concentrando la orina con kaolín, sin ninguna reacción falsamente positiva. Creemos que la variedad de sapos de nuestra región, no presentan los inconvenientes de otros tipos encontrados en otros países y muy especialmente en Estados Unidos. En la Argentina cuya especie es igual a la nuestra, (12) inicialmente se experimentó con hormona foliculoestimulante, estrógenos, benzoato de estradiol, etilbestrol, progesterona, testosterona, desoxicorticosterona, pitresin, tiroxina, prolactina, adrenocorticosterona, cortico suprarrenal, ACTH, adrenalina, ácido ascórbico, acetilcolina, yohimbina, azul de metileno, suspensiones crudas de extractos de hígado, riñón, suprarrenales, bazo, corazón, músculo, estómago e intestino de sapo, orinas de yeguas, ratas, ove- jas, vacas y conejas embarazadas, sin ningún resultado positivo.

\section{En el embarazo ectópico}

Como la implantación corial generalmente es débil, el paso de gonadotrofinas coriónicas a la sangre y posteriormente a la orina es menor que en el embarazo normal, es preciso tratar de inyectar un mayor número de sapos, con mayor cantidad de vehículo y previamente concentrado bien sea con kaolín o hialurinodasa (13) lo que elimina en gran proporción los falsos negativos. La respuesta positiva es de gran ayuda al clínico, pero la negativa no descarta la posibilidad del embarazo ectópico, por la deficiente implantación que puede existir.

\section{En la mola hidatiforme \\ y coriocarcinoma}

La determinación cuantitativa (14) es de gran utilidad en estas entidades, teniendo previamente en cuenta los siguientes factores: Los embarazos normales tienen una eliminación normal que oscila entre 80.000 a 160.000 U.I. pero entre el 45 y 75 día a partir de la última menstruación, se alcanza en un lapso de 24 horas y en forma normal alrededor del día 60 una eliminación que puede llegar hasta 320.000 U. I. de tal manera que una sola determinación en dicho tiempo no hace el diagnóstico de embarazo molar. Igualmente en embarazos gemelares, e hiperemesis gravídica, ocasionalmente se obtiene títulos altos pero en forma esporádica. Dos determinaciones elevadas por encima de 160.000 U. I. y verifi- 
cadas con intervalo de 8 días, sí permiten hacer el diagnóstico de embarazo patológico (15). Después de la expulsión molar puede persistir una reacción positiva entre los 7 días y 6 meses, pero la dosificación cuantitativa verificada cada 15 días en el primer tiempo y cada 45 días en el último, su título creciente o decreciente indica la actividad o regresión de la lesión. En el coriocarcinoma (16), generalmente la eliminación es más acentuada y el límite máximo con la técnica correctamente empleada, no puede ser superior a 1.280.000 U. I., pues corresponde a una gota de orina diluída en 4 cc de agua. Diluciones obtenidas por otros procedimientos pueden dar cifras mucho mayores, pero no deben emplearse ya que esto sólo despista al clínico y no son en manera alguna correlacionadas con las Unidades Internacionales. Para que la reacción cuantitativa tenga todo su valor clínico, debe relacionarse siempre a una sola técnica establecida en forma internacional, ya que varios millones por diluciones arbitrarias, pueden corresponder a un bajo título internacional de diagnóstico.

En los casos debidamente comprobados histológicamente de coriocarcinoma, la persistencia de positividad con títulos cuantitativos crecientes, son de pronóstico fatal a corto plazo.

\section{Variaciones regionales de la reacción}

Los sapos de nuestro medio of recen todas las condiciones ideales para la reacción, pero al igual que las obserervaciones publicadas en los países que tienen estaciones definidas y donde encuentran que en invierno y frío intenso el sapo es poco receptivo o lo hace en un lapso de tiempo al doble o triple del que emplea en el verano, entre nosotros ocurre algo similar y por tal motivo la reacción no es tan rápida en medios de baja temperatura ambiente como lo es Bogotá, y ciudades de similitud climatérica. Por tal motivo, las experiencias en ciudades de clima frío, no son tan halagadoras como las que se encuentan en nuestro clima y similares.

La reacción de Galli comparada con los test inmunológicos y fluorescencia

Esta reacción ideal en nuestro medio por los factores expresados anteriormente, en vista de no haber sido de tanta fidelidad y exactitud en todo el mundo, ha inducido a buscar otra adaptable en todas las condiciones climatéricas. En los últimos cinco años encontramos abundantes publicaciones en todas las revistas ginecológicas de pruebas basadas en la reacción inmunológica entre la gonadotrofina coriónica que actúa como antígeno y un anticuerpo (anti-gonadotrofina coriónica humana) que se encuentra incorporada en una placa de agar. Por diversos medios se puede apreciar la reacción antígeno anticuerpo, como la fluorescencia (17) donde el contacto del anticuerpo gonadotrópico con la orina da fluorescencia a los eritrocitos. Diferentes sistemas basados en el mismo principio (18-19), encontrando en algunas falsas reacciones positivas sobre todo en la administración prolongada de compuestos progestacionales (20) o ingestión prolongada de compuestos sa- 
licilados (21) reacciones ampliamente difundidas, permitieron perfeccionar los diversos procedimientos, hasta llega: a tener hoy en día la reacción ideal, fácil de verificar en dos minutos (22) con respuestas positivas en el $80 \%$ de los embarazos ectópicos (23) y de tanta o mayor seguridad como la reacción de Galli (24).

La reacción que nos ocupa se basa en una hemoaglutinación inhibitoria usando un antígeno de gonadotrofina coriónica con partículas de latex absorbidas, que puestas en contacto con gotas de orina o suero de la paciente que se investiga, produce una aglutinación en caso de que no existan anticuerpos capaces de neutralizar el antígeno (reacción negativa) o una completa penetración del antígeno anticuerpo y sin ningún fenómeno aglutinante, en caso de reacción positiva.

Estudios verificados en Budapest (25) (donde se denomina pregnosticon), Japón (26), Escocia (27), Italia (28) y abundantes publicaciones en Norte América están de acuerdo en considerarla como la reacción ideal, por su fácil ejecución, eliminación del factor biológico, precocidad y seguridad similar a la que nos brinda la reacción de Galli-Mainini.

\section{Resumen y conclusiones}

19 Se analizan los factores básicos en la R. de Galli-Mainini, que son aplicables a los otros tipos de reacciones en el diagnóstico precoz del embarazo, dándole primordial importancia al factor sapo, responsable de la disparidad de conceptos en la reacción, por no ser todos receptivos ni presentar las mismas características biológicas.

20 Es preferible en la reacción la orina al suero, con mejores resultados cuando en la primera no existen factores de dilución (baja densidad). En el feto muerto, su interpretación es muy relativa, en los tumores testiculares no presta utilidad y en nuestro medio no es modificada por la ingestión de tranquilizantes.

$3^{\circ}$ La reacción cuantitativa debe interpretarse conociendo las causas normales de altos niveles y relacionándola siempre a unidades internacionales, sin diluciones caprichosas que dan títulos elevadísimos que confunden al clínico. Es de mucha utilidad en la mola hidatiforme y coriocarcinoma, sobre todo en el pronóstico de este último.

\section{4⿳亠丷 La reacción de Galli-Mainini} está encontrando su reemplazo con la hemoaglutinación inhibitoria con antígeno de gonadotrofina coriónica y partículas de latex absorbidas, por su sencillez, exactitud, precocidad y rapidez en los resultados, según estudios verificados actualmente en Estados Unidos, Europa, Japón, etc. 


\section{BIBLIOGRAFIA}

1. GALLI-MAININI C.: Pregnancy test using the male batriachia. J.A.M.A 138: (121-126). 1948.

2. BRADLEY M. PATTEN: Embriología humana. Edit. El Ateneo, 1956. Buenos Aires.

3. HON E. H. and MORRIS: Further experience with the toad method of testing chorionic gonadotropin in normal disturbed pregnancy". J. Cl. End. Med. 16/10 (1.354-1363). 1956.

4. WIENINGER: Univ. Frauenklin. The Galli-Mainini test in a native toad. Excerpta Med. Obs. Gynec. Vol. 7, 1.369, 1961.

5. FRAZER J. F. D.: The one hour pregnancy test using male British toad. J. Obs. Gyn. Brit. Emp. 64/6 (883-886). 1957.

6. NISHIJINA AYA ISHIDA: Clinical studies of the male frog pregnancy test. Unive. Tottori, Japón. Excerpta Med. Obs. Gyn. Vol. II/8, $1.238,1958$.

7. GALLOSKY F.: Early diagnosis of pregnancy with simultaneous by performed melanophore and Galli-Mainini reaction. Akus. Ginec Abstracts of Soviet Medicine B., pág. 241, 1956.

8. Mc COMARCK G.: A. comparision of the color chemical with Friedman modification of Aschheim-Zondek. Test. Am. J. Obs. Gyn. 46 (522-527). 1951.

9. HODGSON J. E. CHARLES: Accurate pregnancy testing in tranquilized patients. J.A.M.A. 170 (1.890-1892), 1959.

10. BILLHART J. R.: Tranquilizer interference in the rana pipiens chorionic gonadotropin test. J. Obs. Gyn. 14/5 (581-587), 1959.

11. BUENO E. MIGUEL: Tranquilizing drugs and pregnancy test in male batrachia. Sciencie 135: (925-926), 1962.

12. GALLI-MAININI C.: Estudio sobre la acción de la gonadotrofina en el sapo macho. Rev. Soc. Arg. Biológ. 23: (125-128), 1947.

13. VOLA A. and BELTRANO: Elimination of false negatives in the Galli Mainini pregnancy test. Minerva Med. 12/8 (129-135), 1959.

14. RISSE Th.: The diagnostic value of the quantitative Galli-Miainini toad test. Univer. Frankfurt Excerpta Med. Obs. Gyn. 10: 1183, 1957.

15. TOLAND O. J.: Hydatiforme mo'e with hight titer of gonadotropic hormone. Am. J. Obst. Gyn. 42: (502-508), 1944.

16. PERALTA RAMOS A.: Valor de la determinación cuantitativa gonadotrófica en el diagnóstico del coriocarcinoma". Bol. Inst. Matern. 42: (11-17), 1947.

17. QUERCI V. D. ANTONE: Utilization of immunofluorescence in the diagnosis of pregnancy Minerva Med. 84: 1/2, (16-18), 1964.

18. WURZBURG: Univ. Frauenklin. Comparative studies in biological and immunological pregnancy test. Excerpta Med. Obs. Gyn. 18/3, 1904, 1965. 
19. OLSON AM., ADDUCCI J. E.: An evaluation of the latex floculation test in the diagnosis of pregnancy. Am. J. Cl. Path. 39/6, (589-592), 1963.

20. GOLDIN M.: The use de latex particles sensitizied with human chorionic gonadotropin in a serological test for pregnancy". Am. J. Ciin. Path. 38: (335-338), 1962.

21. HENRY J. B. and LITTLE W. A.: Immunological test for pregnancy. J.A.M.A. 182: (230-233), 1962.

22. T. MiALE, T. NOTO, J. MIALE: New inmunological test pregnancy a two minutes slide test. Am. J. Clin. Path. 413: (273-278), 1964.

23. GUSDON J. P.: A 3 minutes pregnancy test. Obst. and Gyn. 23/6, (885-888), 1964.

24. AGÜERO O. and LUGO T.: Reacción inmunológica del embarazo.. Rev. Obst. Gin. (Caracas), 24/2, (383-395), 1964.

25. GOTTINGEN: Univ. Frauenklin (Budapest). The value of a new immunological test for pregnancy. Excerpta Med. Obst. Gyn. 18: 1956, 1965.

26. HANAWA K.: Immunological pregnancy test. Therapeutics Tokyo. Excerpta Med. Obst. Gyn. 18/5, 1.412, 1965.

27. CHARMAN A. and PEARSTON: Immunological test for pregnancy. J. Med. Lab. Thec. Glasgow. Excerpta Med. Obst. Gyn. 18/6, 1.803, 1964.

28. MALEFATLI G.: Comparative and clinical and biological test por pregnancy. Roma. Minerva. Ginec. 16/11 (472-474), 1964.

29. GODTS P. and Mighorst J.: Comparative examination of a serological pregnancy test and the frog test. Am. J. Obst. Gyn. 89/5 (590-594), 1964.

30. CUBONI E.: Le cause d' errore reazioni di gravidanza. Milano Terapia 48: $(362-363), 1963$.

31. YATHIAC: The quantitative toad test in normal and abnormal early gestations. Obs. and Gyn. 23/4 (547-550), 1964.

32. WIRISBURG H.: Univ. Frauenklin. Pregnancy test in frogs at low temperature. Excerpta Med. Obs. Gyn. Vol. 14/10, 1752, 1961.

33. LIU T. L.: (Chinese text). English Abstract. The clinical value of concentrated urine in male toad test. Excerpta Med. Obst. Gyn. Vol. 13/11, 1.936, 1960.

34. MATSUBARA M.: Univ. Tokyo. Matsubara reaction. Excerpta Med. Obt. Gyn. Vol. 17/5, 1.372, 1964.

35. GOVOROV D. A.: (Leningrado). The practical value of Richardson Biochemical test for pregnancy. Excerpta Med. Obst. Gyn. 61: 1,317, 1961. 